\title{
The Economic Effects Of Digital Technology \\ On The Market For Recorded Music
}

Lynde Gilliam, (GilliamL@mscd.edu), Metropolitan State College of Denver

\section{INTRODUCTION}

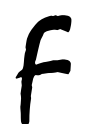

or more than fifty years, the recorded music industry has owed its existence to the development of analog sound recording technology. It was not, however, simply the development of a sound recording technology that created and sustained the industry. It was the fact that this recording technology was analog-based.

Today, music recorded using analog technology is rare, having been almost completely replaced by the recently developed digital recording technology. As the use of digital recording technology continues to increase, particularly in conjunction with the evolving Internet, the likely outcome will be one of significant financial loss and structural change to the traditional recorded music industry.

\section{THE NATURE OF SOUND}

What the human ear perceives as sound is acoustical energy, produced by fluctuating waves of air pressure. Sound air pressure waves fluctuate in cycles, where each complete cycle consists of two half-cycles. The first halfcycle is one of air molecule compression, followed by a second half-cycle of air molecule rarefaction. That is, a sound wave's complete cycle is one of higher air pressure followed by lower air pressure. For a given sound, such as middle $\mathrm{C}$ on a piano, as the intensity of air molecule compression and rarefaction increases, the human ear perceives the sound as becoming louder in volume.

The number of times a sound wave's complete cycle occurs per second (where one cycle per second equals one Hertz, or $\mathrm{Hz}$ ) is referred to as frequency. The human ear is capable of perceiving sound only in the approximate range of $20-20,000 \mathrm{~Hz}$. Capturing these sound waveforms in a manner which can be reproduced is the basis of recording technology.

Until the recent development of digital recording technology, sound was recorded using the only recording technology ever developed. This technology involved the use of different mediums to physically capture a sound waveform analogous in shape (hence the term "analog") to that of the live sound being recorded. By contrast, digital recording technology captures a sound's waveform in a manner that is non-physical. As will be discussed below, this physical versus non-physical distinction in recording technique lies at the heart of the growing uncertainty and instability in the recorded music industry.

\section{THE EVOLUTION OF SOUND RECORDING TECHNOLOGY}

The first technology for recording sound was developed by Thomas Edison in 1877. Edison's technique used a metal stylus to carve a groove into a tin foil-wrapped, rotating cylinder. The particular pattern carved by the stylus was created by sound directed through a horn to a diaphragm connected to the stylus. The resulting physical groove was analogous in shape to that of the live sound waveform being recorded. On playback, this process was reversed. The movement of the stylus in the groove caused the diaphragm to vibrate, emitting sound through the horn. In today's terminology, the horn, in effect, acted as both a microphone and a speaker in recording and playing back sound. 
Originally, Edison believed his sound recording technology would be used primarily for dictation. The public, however, was enthusiastic about the technology being used to produce recordings of live music. In the 1890's, Edison released the first commercial music recordings. Some 20-30 years later, when Emile Berliner substituted discs in place of cylinders as the recording medium, mass-produced commercial recordings became economically feasible. Over the ensuing years, industry standards were developed which ensured consistency in recording formats and technical standards.

By the late 1940's, commercial music recordings had evolved into two disc-based formats: the 45 (so named because the disk rotated at a speed of 45 revolutions per minute) and the LP (for Long Playing disc, which rotated at a speed of $33^{1} / 3$ revolutions per minute). These two formats were industry standards for the next fifty years.

Each of these formats used an analog recording technique which captured live music by physically carving an analogous groove into a vinyl disc. The difference between the two formats was one of physical size, total music which could be recorded, and production costs. The sound quality was the same between the two formats.

The 45 was seven inches in diameter, and could hold approximately 4-5 minutes of music on each side. By the 1950 's, the majority of recorded music purchased by consumers was of the 45 format. Generally, one side of the record (the "A" side) held a single, popular song of 2-3 minutes length. This was the song record producers believed to have the greatest potential for sales. The other side of the record (the "B" side) usually held a lesser-known song by the same artist.

The LP was twelve inches in diameter, capable of holding approximately 20-25 minutes of music on each side. LP's were used primarily for collections of songs (or "albums") by a particular artist.

During this period, professional recording studios used magnetic tape in a reel-to-reel format to create the primary recording of a live performance. This format produced a higher sound quality than did recording directly to the vinyl disc. This magnetic tape recording (known as the "master") was then used to produce vinyl copies in either 45 or LP format for the consumer market.

Recording on magnetic tape rather than vinyl disc is a different format for analog recording. An analogous copy of the live sound waveform is created by imposing patterns onto a magnetic tape. Like recordings on vinyl discs, magnetic tape recordings are a physical construct.

Recorded music using the reel-to-reel format proved inconvenient to the consumer, due to its size and the bulkiness of the playback unit. In 1963, however, Philips introduced a magnetic tape format which was widely embraced by the public: the audio compact cassette.

By the early 1980's, with Philips' development of the compact cassette tape, there were three formats for recorded music available to the consumer: vinyl records, in the 45 and LP formats, and the compact cassette tape .

Around this time, a totally new recording technology was about to be introduced. This digital- rather than analog-based recording technology would change permanently the manner by which live music was recorded. It would also change permanently the basic structure and sustainability of the recorded music industry.

In 1982, digital recording technology was introduced to the market, the result of a joint research project between Sony and Philips. This was the first recording technology to be developed that did not rely on the analog technique. That is, digital recording technology does not rely on creating a physical copy of the sound waveform being recorded. Instead, digital technology captures a sound waveform by "visually" sampling the waveform pattern and storing this data as binary code. In the consumer market, digitally recorded music uses a compact disc (or "CD") format. Approximately $4 \frac{1}{2}$ inches in diameter, a CD is a plastic disc coated with a layer of aluminum and protected with a final layer of lacquer. By the early 2000's, digital recording and CD's had all but replaced analog recording and vinyl records. 


\section{THE ECONOMICS OF LIVE AND RECORDED MUSIC}

Prior to the development of sound recording technology, music could only be experienced in live performance. As such, a single "unit" of the product (i.e., a concert, for example) could benefit more than one individual. Should the concert be held outdoors, the concert producer could not, without incurring significant expense, exclude from benefit those who did not pay for the experience.

A live performance conducted in a concert hall, however, gives the producer the ability to exclude from benefit anyone who does not purchase the product (i.e., a ticket to the concert). Although a significant number of people benefit from a single unit of the product, they do so only if they have paid for that right. In this manner, the public good nature of live music is transformed into a marketable, private good.

With the development of sound recording technology, individuals could experience music in an additional format: recordings of live music. While the recorded music experience differed from that of a live performance, it nonetheless offered benefit to the individual, and a market for the product emerged.

For the emerging recorded music industry, the development of sound recording technology became a doubleedged sword. Sound recording technology offered a new market opportunity in the production and sale of recorded music, or "records".

However, the development of sound recording technology also threatened to undermine the very market it created. That is, consumers now had available a technology by which copies of original records could be created. In principle, a single record could be purchased from its producer and used to create additional copies. As a result, the potential now existed for lower cost copies to be made and sold among consumers, rather than consumers having to purchase copies directly from the producer. To the extent that such copying occurs, there is a loss of sales revenue and profit to the original producer of the record.

In reality, such consumer copying and selling of recorded music was nonexistant in the early stages of the market's development. Several factors explain the absence of consumer copying activity. At the time, copying original recordings was extremely expensive, time-consuming, and required a significant degree of technical expertise. In addition, it required time and energy to locate someone who both owned the specific recording being sought, and was willing to allow the record to be copied. Perhaps most importantly, in the early stages of recorded music, the sound quality of any copy made was extremely poor. Consequently, producers experienced little or no revenue loss due to such copying.

By the early 1980's, refinements in cassette tape recording machines made copying to a cassette tape relatively easy and inexpensive for the consumer. As a result, the potential for lost sales revenue to producers increased as cassette tape recording technology advanced.

Despite such refinements in cassette tape recording, the loss of sales revenue to the recorded music industry due to consumer copying proved to be relatively insignificant. Perhaps the most important copying deterrent was the fact that a cassette tape copy of a 45 or LP record, or another cassette tape, resulted in lower sound quality than the original recording. This reduced sound quality of the copy was not, however, due to the fact the copy was made onto tape rather than vinyl. As is discussed below, the lower sound quality of the copy owed to the fact the copy was made using analog recording technology.

\section{ANALOG RECORDING TECHNOLOGY}

The analog recording process functions by converting sound waves in the air to a physical representation of the waveform on the recording medium. The result is either a groove carved into a vinyl disc, or a pattern of impulses imposed on a magnetic tape. 
Because the analog technique makes a physical copy of the waveform, there is a certain degree of physical contact and resultant "noise" created by the recording process itself. Regardless of the level of isolation and quiet in a recording environment, the analog technique creates noise. As a result, the final recording will be that of a physical waveform which contains both the original sound being recorded, and background noise created by the recording process itself.

Consequently, it is technically impossible to make an exact copy of any recording using the analog process. The first copy, and each successive copy, adds its own background noise, causing signal degeneration and declining sound quality with each copy made. This continuing drop in sound quality is quantified as a decreasing value of the recording's Signal-to-Noise $(\mathrm{S} / \mathrm{N})$ ratio. This consequence of the analog recording technique is the predominant reason wide-scale production of illegal cassette tape copies of original recordings was never more than a relatively insignificant source of lost sales revenue to the recorded music industry. This is evidenced by the fact that, during the period when only analog recording technology was available (i.e., prior to 1982), very few lawsuits were initiated by the recorded music industry for copyright violations.

While the copy's lower sound quality was an important reason consumer copying was fairly insignificant during the analog period, additional factors were involved. As in earlier periods, for example, the technical knowledge required and cost of equipment necessary for copying music were deterrents to many consumers. Also, there existed an information cost to the consumer. As has been noted previously, in order to copy a particular recording, the consumer must locate someone who owns the recording and is willing to have it copied. Permission to copy a recording was not necessarily forthcoming, as the recording process could and did, at times, damage the original recording. In addition, there is a significant time commitment involved in making a cassette tape copy of a recording. For example, copies of recordings were made in "real time": one hour of recorded music required one hour of recording time to copy, during which recording settings needed to be monitored and adjusted as necessary.

Given these deterrent factors, for most consumers it proved easier, less expensive, and resulted in better music quality, to simply go to a store and buy the recording rather than attempting to create a copy. At this point in time, recorded music remained a private, marketable product.

\section{DIGITAL RECORDING TECHNOLOGY}

Digital recording technology is based on a significantly different concept than that of analog recording. Rather than creating a physical image of a sound's waveform, digital recording relies on a non-physical method of replicating the waveform. Because of its non-physical/non-contact technique, there is no background noise created by the recording process itself. This non-physical technology resulted in many of the copying deterrents created by analog recording to be eliminated with digital recording.

In digital recording, the original sound waveform shape (or "amplitude") is captured by sampling the amplitude at intervals of tens of thousands per second, then assigning numeric values to the samples and storing this data as binary code. When the recorded sound is played back, the original sound waveform is reconstructed using the stored data. When a copy of the recorded sound is made, it is numeric data that is being copied. Hence the copy being made is identical to the source being recorded. The only possible variability in the copy as compared to the original recording is found in the sampling rate involved. In the consumer market for music recorded digitally (predominately the CD), the industry standard sampling rate is $44.1 \mathrm{kHz}$. All commercially produced CD's are recorded at $44.1 \mathrm{kHz}$ sampling rate. Equipment necessary for playback of a CD is standardized to playback based on a sampling rate of $44.1 \mathrm{kHz}$. This standard applies today to CD players in home and portable audio systems, car audio systems, and computer CD-ROM drives.

\section{THE ECONOMICS OF ANALOG AND DIGITAL RECORDING TECHNOLOGY}

Overall, between the early 1960's and the early 1980's, consumer copying of recorded material was relatively insignificant. Although such copying is in violation of federal copyright laws, the deterrent effects of such laws are slight. Rather, the minimal amount of consumer copying during this period owed essentially to two factors: the lower sound quality of the copy produced, and the information cost involved in the copying process. 
Following the Sony/Philips 1982 development of digital recording technology and the compact disc, however, consumer copying of recorded music began to increase dramatically. As has been discussed previously, digital recording technology involves a completely different approach to sound recording than used by the analog technique. When digital recording is used, the resulting copy is an exact duplicate of the original. That is, the copy made is not a "good", or "really good" copy of the original; it is an identical copy. Succesive copies made from a copy do not deteriorate in sound quality. A copy made from a copy is identical in every respect to the original recording. In this manner, the development of digital recording technology and the CD format removed one important deterrent to consumer copying of recorded music: copies no longer suffered from lower sound quality.

In addition, other deterrents to consumer copying were significantly reduced or eliminated. For example, there are significant economies involved when comparing the cost of creating a digital copy of a CD with that of purchasing the CD directly from the producer. Today, the average price of a music CD is approximately $\$ 15$. In contrast, the cost of making an identical copy of the CD is approximately $\$ 0.20$.

Also, the time involved in making a copy is significantly reduced, and requires no monitoring from the consumer. A CD containing one hour of music can be copied in less than five minutes. Further, the knowledge necessary for making a CD copy is equivalent to, or less than, that required for making a cassette tape copy. The CD recorder (or "burner") necessary for copying a $\mathrm{CD}$ is standard equipment on today's typical home computer. For older computers with a non-recording CD player (or CD-ROM), the CD player can be replaced by a new CD recorder for less than $\$ 50$.

Finally, with the development of digital recording technology and digital technology in general, consumer copying of recorded music is no longer deterred by the information cost of locating someone with a specific recording and the willingness to allow that recording to be copied. Today, virtually perfect, cost-free information in this regard is available through the Internet. Individuals around the world copy CD's to their computer's hard drive. The hard drive copy is identical to the original CD. These individuals are brought together to share their hard-drive recorded music through numerous peer-to-peer (P2P) websites on the Internet. It is a simple matter for an individual to $\log$ onto one of these websites, search for a specific CD by title (for example, "The Mormon Tabernacle Choir Unplugged"), and find a number of individuals worldwide who have this CD on their computer's hard drive, and are willing to let it be downloaded. In a matter of minutes, an exact copy of the CD will have been downloaded to the individual's computer. At that point, the individual can copy the downloaded songs to a blank CD (a process referred to as "burning" a CD). From the time the individual decided he or she would like to have a CD of "The Mormon Tabernacle Choir - Unplugged", to the time the individual actually has that exact CD in hand, total elapsed time is approximately 15-20 minutes, perhaps less.

\section{IMPLICATIONS OF DIGITAL TECHNOLOGY FOR THE RECORDED MUSIC INDUSTRY}

Since Edison's invention of a technology for recording sound in 1877, "recorded music" has meant music captured and imposed on a physical "thing"; such as a 45 or LP vinyl disc, a cassette tape, or a CD. Given this physical property of recorded music, these "things", or "products", could be produced and marketed as private goods, much like new cars, televisions, or canned beans. Individuals who did not purchase a recording from the producer were denied ownership of the recording. Further, and most importantly, because of significant deterrents to consumer copying of recorded music, the individual had no other options for acquiring the recording.

With the evolution of digital recording technology and the Internet, however, these deterrents to consumer copying of recorded music were eliminated. The existence of these copying deterrents were the critical factors which allowed recorded music to be marketed as a private good. In the absence of these deterrents, recorded music has now become a non-marketable public good. The music on a single CD purchased and uploaded to the Internet can now be downloaded to the hard drive of any computer in the world. In principle, worldwide demand for this CD's music can be satisfied in a matter of a day or two, perhaps less, at a time and financial cost to the consumer of approximately zero. Further, the producers of the original recording ultimately have no ability to prevent such activity from taking place. 
Also, with evolving digital technology and the Internet, the physical "thing" aspect of recorded music will soon disappear entirely. Music increasingly will become encoded binary data, transferred and reassembled into audible sound via the Internet. Eventually, any music represented as binary data in the digital domain will instantly become part of freely available information on the Internet. Attempts to produce and market this music on a physical medium, such as a CD, will fail. The product "recorded music", in any format, is quickly and inevitably becoming obsolete.

Recently there has been a significant increase worldwide in music downloading activity. Given that such downloading activity is almost always in violation of federal copyright laws, it is understandable why the recorded music industry has increased its filing of copyright infringement lawsuits. Prosecuting such violations of copyright law, however, is difficult, time-consuming and expensive for the recorded music industry.

While such legal actions are, on the surface, understandable, they are nonetheless misguided and wasteful of the industry's resources. Attempting to stop or significantly reduce music downloads worldwide is potentially the recorded music industry's Myth of Sysyphus: its efforts could go on indefinitely, ultimately having little or no impact, save that of bankrupting the industry. Rather than attempting to forestall the inevitable, the recorded music industry would be better served were it to allocate its resources to exploring new opportunities presented by evolving digital technology and the Internet.

\section{REFERENCES}

1. Camus, Albert, The Myth of Sysphus and Other Essays, trans. By Justin O’Brien, Vintage (1983, 1955).

2. $\quad$ Davis, Gary and Ralph Jones, Sound Reinforcement Handbook, Hal Leonard Corp., 1989.

3. Gilliam, L, When the Gap Went Online, T-Shirts Didn't Become Free, Proceedings of the International Applied Business Research Conference, article \#357, March, 2004.

4. Hamilton, Marci A., Why Suing College Students for Illegal Music Downloading is Right, www.cnn.com.

5. Legon, Jeordan, CDs May Soon Go the Way of Vinyl, CNN, September 8, 2003, http://www.cnn.com/2003/TECH/ptech/09/03/cd.future/index.html.

6. MacMillan, Robert, Internet Sparks a Copyright Fire, washingtonpost.com, June 24, 2003.

7. History of Recordings, Recording Industry Association of America, http://www.riaa.com/issues/ audio/history.asp, 2003

8. CNN/Money, October 8, 2003, http://www.cnn.com/2003/TECH/ptech/10/08/bmg.protection.reut/index.html

9. CNN/Money, October10, 2003, http://www.cnn.com/2003/TECH/biztech/10/10/

bmg.protection.reut/index.html 